\title{
BMJ Open Reopening strategies, mobility and COVID-19 infections in Europe: panel data analysis
}

\author{
Jeffrey Franks, Bertrand Gruss, Carlos Mulas-Granados, Manasa Patnam (i) , \\ Sebastian Weber
}

To cite: Franks J, Gruss B, Mulas-Granados $\mathrm{C}$, et al. Reopening strategies, mobility and COVID-19 infections in Europe: panel data analysis. BMJ Open 2022;12:e055938. doi:10.1136/ bmjopen-2021-055938

- Prepublication history and additional supplemental material for this paper are available online. To view these files, please visit the journal online (http://dx.doi.org/10.1136/ bmjopen-2021-055938).

Received 29 July 2021

Accepted 16 November 2021

Check for updates

(c) Author(s) (or their employer(s)) 2022. Re-use permitted under CC BY-NC. No commercial re-use. See rights and permissions. Published by BMJ.

European Department, International Monetary Fund, Washington, DC, USA

Correspondence to Dr Manasa Patnam; mpatnam@imf.org

\section{ABSTRACT}

Objectives Characterise the reopening policies of European countries after the first wave of infections and evaluate how these policies affected economic activity and subsequent infections.

Study design Using publicly available data, we construct a database of reopening policy announcements by country authorities and develop measures related to the speed and timing of reopening. Using panel data regressions, we then assess how a country's reopening action subsequently affected its mobility and COVID-19 infections. Samples of 22 European countries used in the study comprise: Austria, Belgium, Czech Republic, Denmark, Finland, France, Germany, Greece, Ireland, Israel, Italy, Netherlands, Norway, Poland, Portugal, Romania, Russia, Spain,

Switzerland, Turkey, Ukraine and the UK.

Main outcomes Mobility index as well as COVID-19 case and death counts.

Results Reopening policies are associated with a 1.5 percentage point increase in mobility and a $4 \%$ increase in subsequent infections after 2 weeks. However, some reopening strategies are associated with lower infection risk. In particular, early and fast reopeners saw 5\%$10 \%$ increases in infections relative to those that opened later and adopted a gradual approach. The sequencing of sectoral reopenings matters, with infection amplification effects larger for some sectors (like retail and events) than others (like schools).

Conclusions Findings suggest some merit of gradual and late reopening strategies with a careful sequencing of sectoral openings based on their infection amplification risks.

\section{INTRODUCTION}

Europe was among the regions most severely affected by the coronavirus (COVID-19) in the early months of 2020. The escalation of cases during the first wave of the pandemic led governments to introduce stringent lockdown measures in order to slow the spread of the virus and avoid overwhelming the health sector. ${ }^{1}$ When the first wave of infection curves flattened and the outbreak appeared controlled, most European countries started to reopen their economies to alleviate the unprecedented economic contraction generated by the lockdown. ${ }^{2}$ However, the

\section{Strengths and limitations of this study}

- Provides an analysis of exiting from COVID-19 lockdowns in Europe, based on compiling a new database covering the sectoral reopening policies taken in Europe after the first wave of infections.

- Leverages the most disruptive and widespread 'natural experiment' of mobility restrictions during the first lockdown to explore which types of postlockdown reopening strategies are effective and safe in terms of reducing infection risk.

- Constructs novel metrics on the time, speed and sequence of reopening. This allows to link observable measures of infection risk with the overall reopening strategies. The findings can help guide policy on how sectors can be phased for reopening.

- Results on sectoral sequencing do not incorporate granular data, such as on person-to-person infection transmission, or other considerations such as social equity.

- Does not assess the full extent of the trade-off between health and economic outcomes, which would involve a joint analysis of an array of other factors such as economic support policies as well as the social costs of containment measures.

strategies adopted to reopen the economy differed significantly across countries in terms of timing, pace and sequencing of sectoral reopening measures. The crosscountry differences in the evolution of infections following reopenings are also notable.

While there is, by now, a large literature on how lockdown affects activity and infections, ${ }^{3-7}$ there is relatively less evidence on the effects of reopening policies. Several studies have proposed optimal strategies to exit from lockdowns. ${ }^{8-10}$ However, to our knowledge, there is limited ex-post assessment of how different reopening strategies fared, especially in terms of containing infection spread. Given this, the aim of the paper is to analyse how the de jure reopening measures translate into de facto improvements in activity and how they influence the subsequent evolution 
of COVID-19 infections. The paper also explores whether some reopening strategies are associated with lower infection risks than others and, if so, whether it comes at the cost of subdued economic activity.

Our paper contributes to the literature on pandemicrelated reopening policies and its effect of economic activity and infections. A previous study ${ }^{11}$ qualitatively examines the approaches taken by nine high-income countries that eased COVID-19 restrictions and finds that countries diverged in terms of the speed, scale and intensity at which they have implemented easing. A closely related paper ${ }^{12}$ uses mobility and case data to model the effect of coordinated exit strategies in Europe and finds that infection risks are higher when well-connected countries end their interventions prematurely. Our paper documents similar results but by undertaking an empirical analysis of observed outcomes, analysing both early and fast reopening strategies jointly as well as the optimal sequencing of sectoral reopenings.

Two other studies ${ }^{13}$ analyse reopening policies across states in the USA and find that mobility increases a few days after the policy change and that a third of the increase is attributable to the reopening policies. Two related studies from the USA ${ }^{1516}$ distinguish the impact of government policies on lockdown/reopening from people's voluntary behavioural response. The latter effect can materialise if people choose to voluntarily stay at home or resume activity regardless of the restrictions imposed or relaxed, based on their perception of infection risk. While there is mixed evidence on the two different effects, our results suggest that, on average, reopening policies explained the bulk of mobility activity after reopening with a limited role played by voluntary behaviour and that cases increased following reopening. However, we show that these effects disproportionately affected countries that reopened fast and early.

To the best of our knowledge, our paper is among the first to compile data on sectoral reopening policies in Europe and construct metrics on the speed of reopening. A related paper on sectoral containment measures ${ }^{17}$ examines the effect of lifting non-pharmaceutical interventions in over 131 countries on the time-varying reproduction number $(\mathrm{R})$. They find a significantly increasing trend over time in the $\mathrm{R}$ ratio following the relaxation of school closure and bans on public gatherings. In contrast to their study, our paper documents how the increase in directly observable measures of infection risk (such as cases and deaths) depends crucially on the overall strategy of reopening, specifically its timing and speed. We are also among the first to document how the sectoral effects vary by the phase in which it reopened, allowing us to compare infection amplification risks for each sector.

\section{METHODS}

\section{Reopening database and other data sources}

During the reopening that followed the first wave of the pandemic, most European economies took a phased approach, opening sectors differentially and in a gradual manner. We construct a database ${ }^{18}$ of government reopening policies that were announced to exit from the first wave of lockdown. More precisely, we compile the measures taken by the authorities of 22 European economies to reopen the economy based on the (1) sector, (2) timing, (3) phase, and (4) intensity of reopening. For each country and date, we define the reopening measures as follows:

1. Sector of reopening: sectors are classified as schools, industry, retail, services (eg, hotels, restaurants, hairdressers, etc), events/public places and international travel (including intra-European).

2. Timing of reopening: the date and phase in which a country opened a specific sector

3. Phase of reopening: the associated sequence in which a sector was opened as part of the overall exit plan.

4. Intensity of reopening: change in the opening status of a particular sector. Opening status is coded as 0 (open), 1 (open with restrictions/guidelines), 2 (partially open with only a subset of the sectors allowed to function) and 3 (closed).

These indicators are constructed based on governments' reopening measures from official and other news sources. The paper also uses supplemental information from the Oxford COVID-19 Government Response Tracker, ${ }^{19}$ the European Commission measures dashboard $^{20}$ and the ACAPS government measures data set. ${ }^{21}$ In comparison to other existing data sets that capture the level of government containment measures and their stringency, our data capture the sector-specific targeting of reopening measures.

We use two other key data sources for measuring our two main outcome variables of interes - economic activity and infections. For the first outcome on economic activity, we use a high-frequency proxy-mobility data provided by Google. ${ }^{22}$ The Google mobility measure captures movement trends by country across different categories of places. The data show how visitors in (or time spent in) categorised places change compared with baseline days. A baseline day represents a normal value for that day of the week. The baseline day is the median value from the 5-week period of 3 January- 6 February 2020. For our analysis, we use the average mobility across three main mobility categories: retail and recreation, public transport and workplaces. We omit other categories related to parks, supermarkets and residential as they are less likely to reflect economic activity. They are also less affected by containment measures. The changes in our overall mobility measure track the changes in Gross Domestic Product (GDP) fairly well, with an estimated correlation coefficient of about 0.5 in 2020 Q2 explaining over $70 \%-80 \%$ of GDP variability.

As the Google mobility index uses the extent of mobility in January 2020 as baseline, there could be seasonal effects contaminating our analysis, especially during the summertime period. To account for this, we use the TomTom Traffic Index ${ }^{23}$ as an alternative mobility indicator in robustness exercises. This index does not suffer from seasonality problems as it shows, for each country, 
the difference in average congestion levels in 2020 with respect to the same period in 2019.

On the second outcome variable of interest, we use data from the European Centre for Disease Prevention and Control $^{24}$ and Our World in Data ${ }^{25}$ on daily confirmed cases, deaths and tests conducted. We also supplement the health data by using survey data on people's responses to the coronavirus pandemic, conducted by Imperial College London in partnership with YouGov. ${ }^{26}$ The survey measures self-reported intensity of wearing masks and number of social contacts met among other things. See online supplemental appendix section I and online supplemental figures 1-4 for further discussion on trends in mobility, infection and health behaviours during the time period analysed in the study.

The analysis considers policy actions taken between 1 April and 15 July. The reintroduction of restrictions thereafter is not included as this would confound our measure of reopening which relies on easing of restrictions. For the mobility analysis, we also restrict the period up to 15 July, excluding therefore the time around summer vacations so as not to confound the decline in mobility with changes in reopening policies or infections. In a robustness exercise, we extend our analysis to cover the summer holiday period using our alternative measure of mobility (TomTom) which is seasonally adjusted.

\section{Characterisation of reopening plans}

Using our data set of reopening we calculate two related metrics that help characterise the overall reopening plan for each country: the speed and timing of reopening plans.

- The timing of a country reopening strategy is calculated as the percentage change in daily deaths between the peak of the infection-death curve and the day the first reopening measure is introduced. This measure correlates highly with the number of days a country spent in its first lockdown and can be loosely interpreted as such.

- The speed at which countries reopened is computed as the ratio of effective to actual days since the first reopening measure is introduced. The effective days open is defined, at the sector level, by the cumulative extent of each sector's reopening each day. For instance, if a country reopened schools for 4 days by $50 \%$ and subsequently by $100 \%$ for 1 day, the effective days reopened for schools is 3 . The aggregate effective days open is the sum of the effective days open across all sectors.

A first clear difference relates to the timing of the first reopening measures in relation to the epidemiological situation in the country (figure 1A). Using the evolution of daily fatalities as an indication of the stage of the pandemic, the data reveal that some countries (eg, Belgium, France) only started opening when the number of daily deaths had declined by about $80 \%$ with respect to the peak attained before; other countries, instead, opened around the time fatalities started to decline (eg,
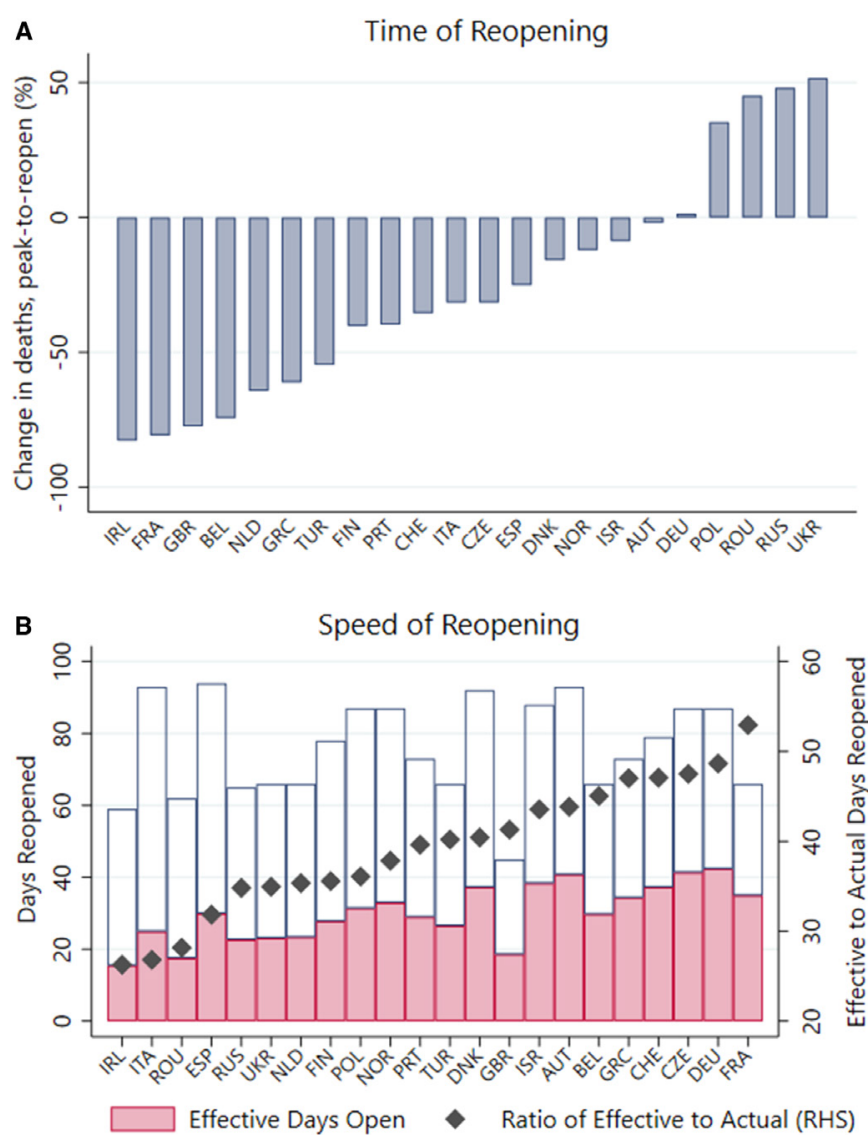

Figure 1 The graph in the top panel A plots the growth of daily deaths from peak to reopen. Note that the sign for the peak to reopen change in death is reversed for countries that open before the peak is reached. The graph in the bottom panel $\mathrm{B}$ plots the number of days since reopening for all countries in the database. The shaded area shows the number of effective days open which is measured as the cumulative (daily) extent of reopening across sectors. The diamond markers show the ratio of effective to actual days open. AUT, Austria; BEL, Belgium; CHE, Switzerland; CZE, Czech Republic; DEU, Germany; DNK, Denmark; ESP, Spain; FIN, Finland; FRA, France; GBR, UK; GRC, Greece; IRL, Ireland; ISR, Israel; ITA, Italy; NLD, Netherlands; NOR, Norway; POL, Poland; PRT, Portugal; ROU, Romania; RUS, Russia; TUR, Turkey; UKR, Ukraine.

Austria, Germany) or even when they were still on the rise (eg, Poland, Russia).

Another key difference across country plans relates to the speed or pace of sectoral reopening actions once they started opening (figure 1B). One way of capturing the difference in speed is to compute, at any given time, the ratio of effective days open (taking into account the extent of easing actions introduced at the individual sector level) to actual days open since the first reopening measure is introduced. As of mid-July, when reopening plans had plateaued, this metric ranged from around $30 \%$ (eg, in Italy and Spain, which followed a gradual approach) to above $50 \%$ (eg, in France, which opened at a fast pace once it started).

Our database also provides us with measures on the sequencing of sectors as they reopened. As countries 
A Sectoral Reopening Sequence

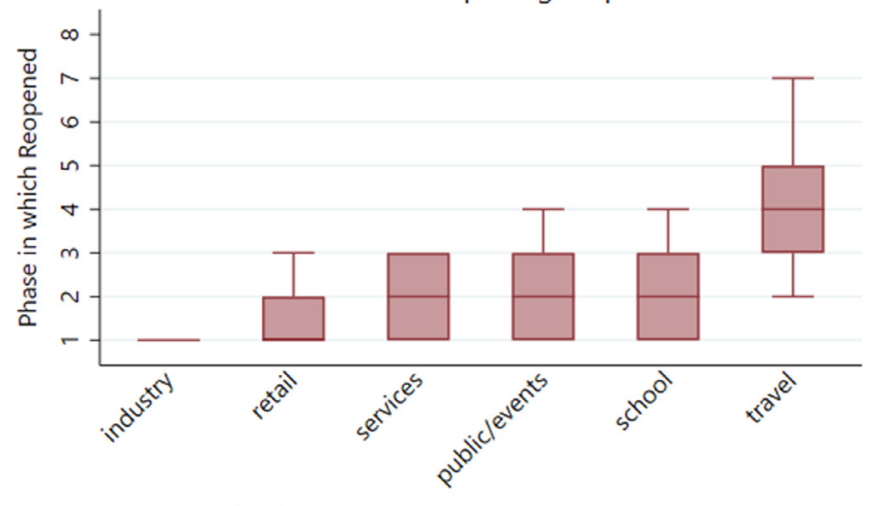

excludes outside values

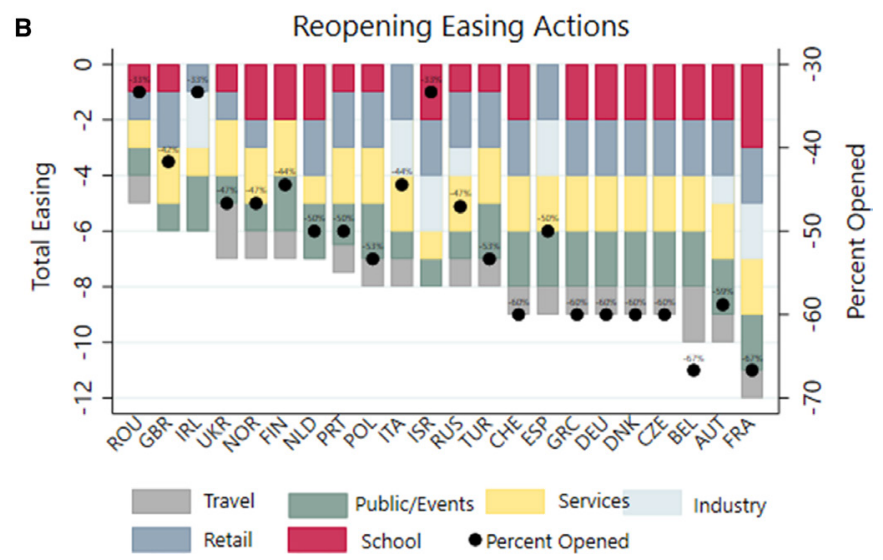

Figure 2 The graph in the top panel A is a boxplot which shows the distribution, across countries of the phase in which each sector opened. The graph in the bottom panel B plots the cumulative changes in the reopening index as of 30 June. The index ranges from 0 to 18 based on the opening status of school, retail, service industry, public/events, travel with 0 (open), 1 (open with restrictions), 2 (partially open), 3 (closed); dots show per cent of restrictions removed. AUT, Austria; BEL, Belgium; CHE, Switzerland; CZE, Czech Republic; DEU, Germany; DNK, Denmark; ESP, Spain; FIN, Finland; FRA, France; GBR, UK; GRC, Greece; IRL, Ireland; ISR, Israel; ITA, Italy; NLD, Netherlands; NOR, Norway; POL, Poland; PRT, Portugal; ROU, Romania; RUS, Russia; TUR, Turkey; UKR, Ukraine.

announced their reopening plans, they typically did so in phases that featured the reopening of different sectors. Figure 2A shows, on average, industries were among the first to reopen, while retail and services were featured mostly in phase 2. Schools and international travel were often among the last to reopen, although in some countries schools did open in the first phase. Figure 2B shows the significant amount of variability across sectors and countries in easing actions. The figure plots the total extent of easing restrictions by sector on the left-hand side axis and the average fraction of restrictions eased on the right-hand side axis. By the end of June, countries had eased $33 \%-66 \%$ of their lockdown restrictions by easing a range of restrictions across sectors.

\section{Study design and statistical analysis}

The paper estimates the effect of reopening on activity and on infection-related variables using panel data of daily observations for 22 countries. As there is considerable uncertainty regarding the timing of the effect of reopening, which can be reflected in non-linearities in the response of economic activity and infections to reopening measures, we use projection methods. ${ }^{27}$ This approach provides a simple and flexible framework that is robust to misspecification by fitting a separate regression for each horizon of interest $h$, without imposing smoothness in the impulse response across horizons (as is the case under a traditional autoregressive approach). It also allows to accommodate estimation of interaction effects in the impulse responses. In our application, we compute the effect of reopening at a given time, on infections and mobility, 7-30 days ahead. Our estimation equation is thus:

$$
\begin{aligned}
Y_{i, t+h}= & \alpha_{i}^{h}+\eta_{t}^{h}+\beta^{h} \cdot \text { Reopening }_{i, t}+\lambda_{1}^{h} \cdot \text { Deaths }_{i, t-1^{+}} \\
& \lambda_{2}^{h} \text {. }^{+} \text {ases }_{i, t-1}+\mu^{h} \text { Mobility }_{i, t-1}+\theta^{h} \cdot \mathbf{X}_{i, t}+u_{i, t}
\end{aligned}
$$

The main outcome variables, $Y_{i, t+h}$, are a mobility index and COVID-19 infections (cases and deaths). For the mobility index, the Google mobility data are realigned in index form, where 100 corresponds to baseline (normal) mobility with values below 100 indicating the percentage mobility below normal. The data for infection (daily cases and deaths per million) are defined as the log of the 7-day moving average of daily deaths and daily cases per million, respectively. Our explanatory variable of interest, Reopening ${ }_{i, t}$, is the cumulative easing (reopening) action summed across all sectors. $\mathbf{X}_{i, t}$ is a vector of covariates including a control for the first reopening, country infection trends and day of the week effects. All specifications also include a full set of country and time fixed effects $\left(\alpha_{i}\right.$ and $\left.\eta_{t}\right)$. Equation 1 is estimated by Ordinary Least Squares (OLS) for each daily horizon $h=1, \ldots, H$. Standard errors are robust to heteroscedasticity and serial correlation (using a bandwidth of 7 days). All analyses were performed using the STATA statistical software. ${ }^{28}$

The variance decompositions reported are derived by decomposing the share of explained variance of the model in equation 1 into contributions of regressor variables using the Shapley value method.

Analysing the effect of containment policies is potentially subject to endogeneity concerns, and the estimates could be biased if time-varying unobservables affect both mobility and reopening plans. Including lagged infections and mobility in the baseline specification attenuates this concern but may not be sufficient. In the paper, we perform several sensitivity analyses to address this concern (see the Sensitivity analysis section).

We should note that while the first round of containment measures was typically accompanied by an introduction of other policies simultaneously (eg, social distance guidelines, unemployment schemes that encouraged workers to stay at home), this was less so for the 


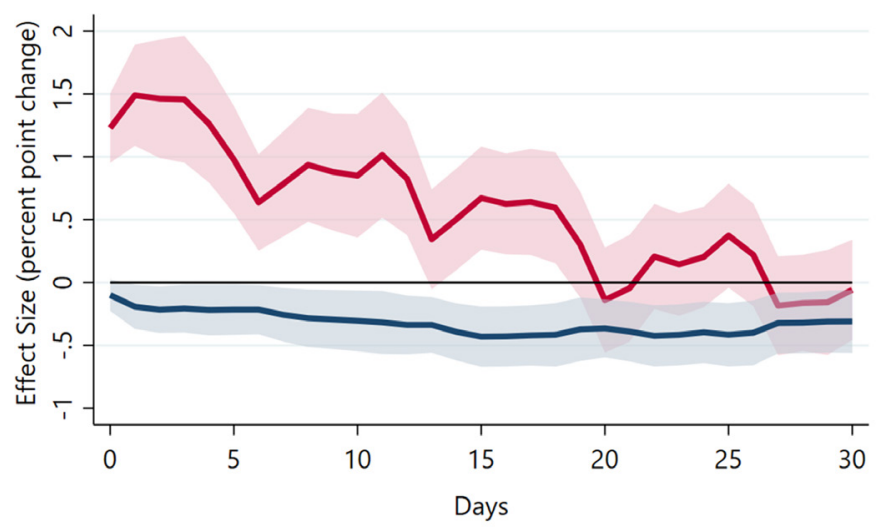

Effect of Reopening Index Effect of Voluntary Distancing

Figure 3 The graph shows point estimates (solid line) of reopening measures and lagged daily deaths on mobility with $90 \% \mathrm{Cl}$ (shaded area) adjusted for heteroscedasticity and autocorrelation.

case of reopening. This allows us to isolate more clearly the reopening policy action from other policies as the announcement of emergency measures typically faded out during the reopening period.

\section{Patient and public involvement}

There was no patient or public involvement.

\section{RESULTS}

\section{Effect of reopening policies on mobility}

We start by assessing the effects of aggregating reopening policies on overall mobility. We find that, conditional on the first reopening action, the marginal effect of a one unit of reopening (or easing restriction) is associated, on average, with an initial increase in mobility of 1-1.5 percentage points (figure 3 , red line). The effect declines gradually over time but remains statistically significant for almost 2 weeks. Detailed results for select horizons are reported in online supplemental table 1.

Changes in mobility can reflect the effect of policies but also the role of voluntary behaviour if people perceive the risk of infection to be low based on tracking daily cases/ death and recommence their activity. Figure 3 (blue line) plots the effects of lagged infection (measured as the average number of daily deaths over the preceding week) on mobility, which can be roughly interpreted as the effects of voluntary reduction in mobility in response to infection spread.

We find that, conditional on the first reopening action, the marginal effect of a unit increase in per-capita daily deaths is associated with a decline in mobility of up to 0.5 percentage point. This effect increases across horizons and remains statistically significant. In terms of the relative magnitude of the two factors, the results suggest that policy actions explain a larger fraction of the increase in mobility than what can be attributed to voluntary behaviour. For instance, a 1 standard deviation increase in reopening leads to a rise in mobility by 0.2
Table 1 Explained variation in mobility (2-week horizon)

\begin{tabular}{ll}
\hline Variable & Per cent variation explained \\
\hline Lagged mobility & 27.08 \\
\hline Reopening & 30.68 \\
\hline / of which: & \\
\hline School & 2.91 \\
\hline Retail & 6.70 \\
\hline Industry & 1.92 \\
\hline Services & 6.49 \\
\hline Travel & 1.67 \\
\hline Public/events & 4.21 \\
\hline Overall reopening period & 6.75 \\
\hline Infections & 16.62 \\
\hline of which: & \\
\hline Cases & 6.91 \\
\hline Deaths & 9.70 \\
Time trend & 25.61 \\
Overall variation explained & 67.3 \\
\hline
\end{tabular}

standard deviation, while a 1 standard deviation decline in daily deaths per million is associated with an increase in mobility of only 0.05 standard deviation.

Another way to assess the relative importance of reopening policies versus lagged infections (or voluntary behaviour) in explaining changes in mobility is to compute the explained variation in the dependent variable that can be attributed to the different regressors. In order to do this, we follow the Shapley method ${ }^{29}$ to decompose the relative contribution of each variable in our specification in explaining the overall variability in mobility. The results are reported in table 1.

We find that, at a 1-week horizon, the model explains about $67 \%$ of overall variability in mobility. The bulk of the explained variation (about $31 \%$ ) is attributed to the reopening policies. Within reopening policies, the reopening of retail and services accounts for the largest share (around 6\% each), followed by events (about $4 \%$ ). Lagged infections or voluntary behaviour explains a much smaller fraction (16\% of overall explained variation). The rest is explained roughly equally by lagged mobility and the country-specific infection trend variable.

The result that voluntary behaviour matters less than reopening policies is in line with the model suggested by Glaeser et al, ${ }^{15}$ which shows that easing restrictions can signal that going out has become safer. Government actions, therefore, have both a direct effect (preventing people who want to go out from doing so) and an indirect effect (signalling to people when it is safe to go out again).

\section{Effect of reopening policies on subsequent infections}

A key question to assess the success of reopening strategies is whether they carry a significant increase in infection 


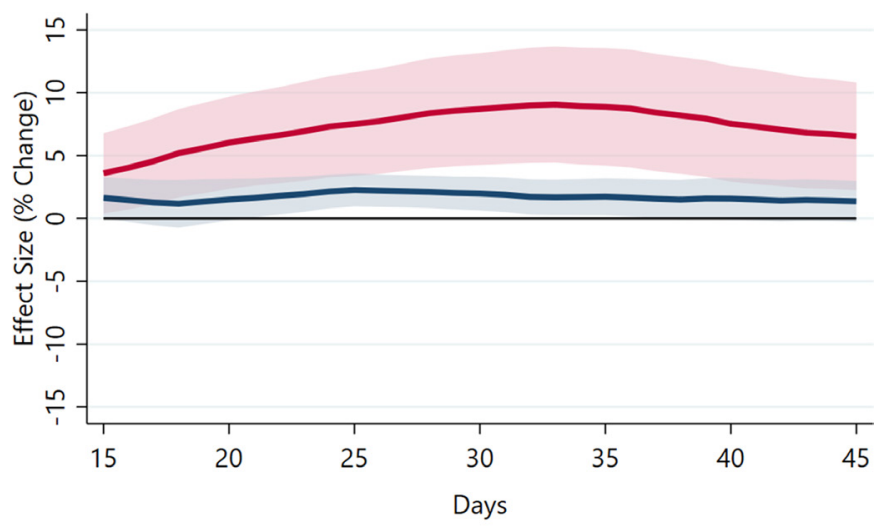

_ Effect of Reopening on Daily Cases

Effect of Reopening on Daily Deaths

Figure 4 The graph shows point estimates (solid line) of reopening measures on the (log) 7-day moving average of cases and deaths with $90 \% \mathrm{Cl}$ (shaded area) adjusted for heteroscedasticity and autocorrelation.

risk. To answer this question, we start by exploring the average effect of reopening measures on infections. For this analysis, we use both the log of daily confirmed cases and deaths as an outcome variable. By estimating the reduced form effect of reopening policies on infections, we are implicitly allowing reopening measures to have an impact on infections via the increased mobility associated with that policy but also through other sector-specific implications for social distancing.

Figure 4 shows the estimates with the red line showing the effects of a unit easing associated with reopening on daily cases over the subsequent 2 weeks, while the blue line shows the results for daily fatalities. The results suggest that a unit easing in the aggregate reopening index is associated, on average, with a significant increase of about $4 \%$ in daily cases after 2 weeks and close to $8 \%$ after 1 month. The effect for fatalities is also statistically significant but quantitatively smaller: daily deaths increase by about $2 \% 1$ month after each unit of easing. Detailed results for select horizon are reported in online supplemental tables 2 and 3 .

In contrast to studies that focused on lockdown measures, ${ }^{30}$ we find a much lower magnitude for the effects of containment policies on deaths. The smaller response of fatalities during reopening, compared with that experienced during lockdowns, could reflect, among other factors, a shift in the demographics of the infected population towards a more younger age group, and the expansion of testing which led to an increased detection of cases.

To assess the relative importance of reopening policies versus the intrinsic dynamics of the epidemic, we decompose the relative contribution of each variable in explaining the overall variability in infections using the Shapley method. The results are reported in table 2.

We find that, at a 3-week horizon, the model explains about $45 \%$ and $80 \%$ of overall variability in cases and deaths, respectively. A sizeable share of explained
Table 2 Explained variation in infection (3-week horizon)

Per cent variation explained

\begin{tabular}{|c|c|c|}
\hline \multirow[b]{2}{*}{ Variable } & \\
\hline & Cases & Deaths \\
\hline Lagged mobility & 2.39 & 10.66 \\
\hline Reopening & 24.50 & 20.27 \\
\hline \multicolumn{3}{|l|}{ of which: } \\
\hline School & 1.58 & 1.69 \\
\hline Retail & 1.59 & 4.77 \\
\hline Industry & 4.04 & 1.79 \\
\hline Services & 0.97 & 2.45 \\
\hline Travel & 2.01 & 1.01 \\
\hline Public/events & 3.36 & 3.11 \\
\hline Overall reopening period & 10.93 & 5.42 \\
\hline Infections & 71.11 & 55.89 \\
\hline \multicolumn{3}{|l|}{ of which: } \\
\hline Cases & 59.63 & 22.74 \\
\hline Deaths & 11.58 & 33.14 \\
\hline Time trend & 1.86 & 13.16 \\
\hline Overall variation explained & 45.05 & 80.69 \\
\hline
\end{tabular}

variation (about 20\%-24\%) is attributable to the reopening measures. However, an even larger fraction of the variability in subsequent infections (around $69 \%-73 \%$ ) is attributable to the dynamics of the epidemics itself, captured by lagged infections and the country-specific infection trend variable. Lagged mobility accounts for a small share $(3 \%-10 \%)$ and could pick up the effect of both lagged reopening policies (over and above what is explained by the current reopening policies) and voluntary movements.

\section{Are some reopening strategies riskier in terms of subsequent infections?}

We next explore whether the average positive effect of reopening on subsequent infections can be attributed to certain reopening strategies (ie, if they are driven by countries that adopted more ambitious reopening plans). In particular, we explore whether the effects of reopening on daily cases and deaths vary depending on the speed of countries' reopening strategies and on the sequencing of sectoral measures.

\section{Do faster and earlier reopening strategies carry more risk?}

To explore the role of the pace of reopening strategies, we classify countries into fast and slow reopeners based on the speed and timing metric (computed as the ratio of effective to actual days reopened and the change in deaths per capita from peak to reopen). The baseline regression is extended to include two indicator variables classifying each country into whether they were fast reopeners (vs slow) and whether they were early reopeners (vs late). We classify a country as an early (late) reopener when the 
A
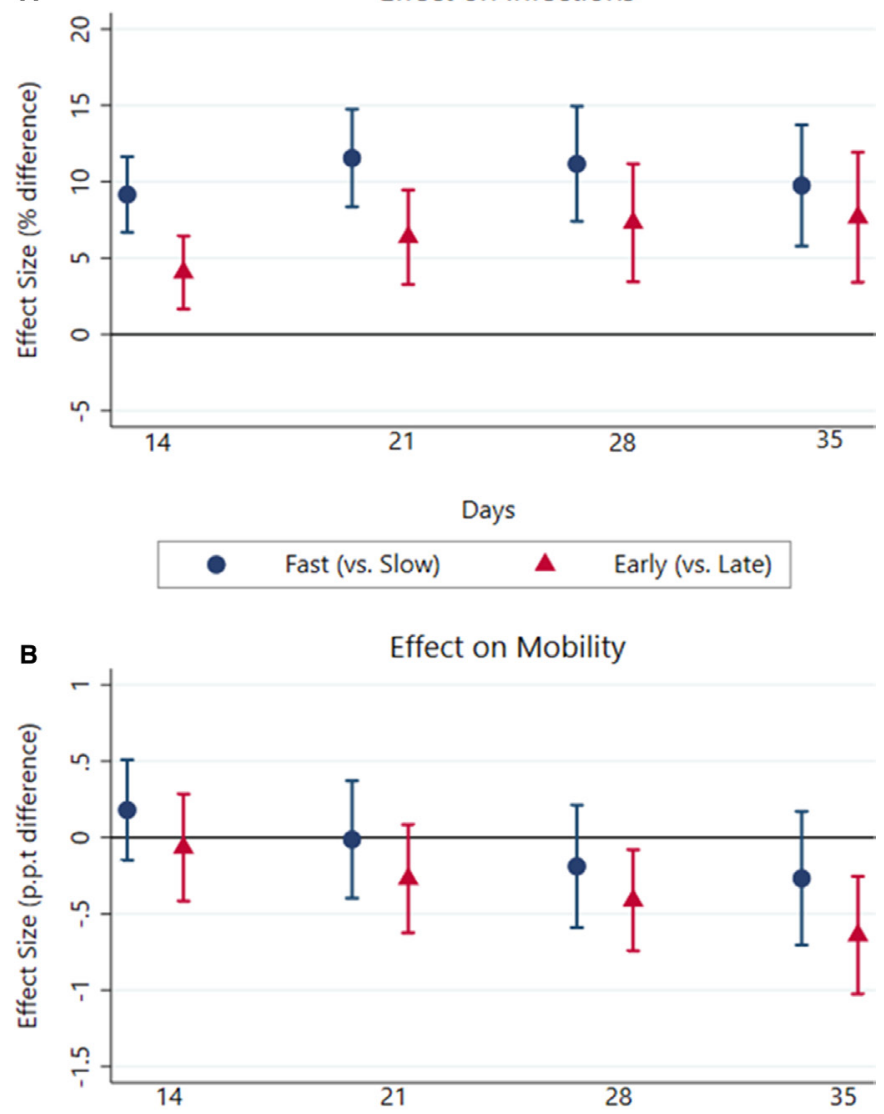

Horizon

Fast (vs. Slow)

A Early (vs. Late)

Figure 5 The graph in the top panel A shows the percentage difference in daily cases between fast and slow reopeners (blue dot with $90 \% \mathrm{Cl}$ line) and early versus late reopeners (red triangle with $90 \% \mathrm{Cl}$ line). The bottom panel $\mathrm{B}$ shows the same differential for mobility; in panel $B$, the $y$-axis shows the effect size in percentage point (p.p.t).

reduction in daily deaths (relative to the peak attained) it had registered before taking the first reopening action is below (above) the median across countries. For countries that registered more than one wave of daily deaths, the first wave is considered. We classify a country as a fast (slow) reopener when its effective to actual days open, or the speed metric defined previously, is above (below) the sample median. These variables are then interacted with the main variable of interest, the reopening policy to retrieve the differential effects (see online supplemental appendix section II for further details on the methodology).

Figure 5 plots the differential effects of being a fast or early reopener on daily cases and mobility. The results suggest that in countries which pursued a fast reopening strategy, reopening actions had significantly higher daily cases per unit of easing (almost 10\%-15\% higher), relative to countries classified as pursuing a slow reopening strategy. Similarly, countries that opened later along the infection curve also had 5\%-7\% higher daily cases per unit of easing, relative to counties that opened earlier.

Interestingly, for every unit of easing, we do not find any statistically significant mobility differential between fast and slow or early versus late reopeners. This seems to suggest that the effect of reopening on mobility does not vary across reopening strategies. However, in terms of calculating the trade-off, we should note that fast and early reopeners would have experienced a longer period of increase in average mobility uptick relative to slow and late reopeners, despite seeing no difference in the rate at which mobility picked up at reopening.

One concern with our chosen characterisation of early versus late reopeners is that it could induce some mechanical correlation in our infection risk estimates as reopening early or later is implicitly a function of a country's (lagged) infection curve. To ensure that our results are not contaminated by this dependence, we use as an alternative measure the number of days spent in lockdown to classify early versus late reopeners as a robustness check. The results shown in online supplemental figure 5 are broadly unchanged.

One way to illustrate the contrast in the results for mobility and infections is to compare the model predictions for these variables under different strategies. The results indicate that alternative reopening strategies produced marked differences in the trajectory of infections but only minor differences with respect to mobility pick-up (figure 6). In other words, easing containment restrictions by a unit delivers similar economic effects regardless of how and when a country exits, but has differential effects on infections, with a much smaller increase in cases if reopening is pursued in a late and slow manner. In addition, online supplemental figure 6 plots the mobility and infection path of the actual data, showing very similar trajectories as in figure 6 , indicating that the model provides a very good fit for the outcome analyses.

These results do not mean the effect on activity is inconsequential since postponing or slowing the reopening actions implies that full reopening is delayed. But the results suggest the economic cost of gradual strategies is not disproportionately larger, while the infection risk appears to be so.

We also explore how the extent to which a country's reopening strategy affects infections depending on other countries' reopening. A priori, infection amplification risks could be higher for early and fast reopeners when other countries have already reopened their economies significantly, for instance, through the international travel channel, or, as it may signal that it is safe to relax social distancing practices. We analyse this by further interacting the early (vs late) and fast (vs slow) effects with the fraction of reopening achieved in peer European countries. The results in figure 7 suggest that the infection risk of early and fast reopening strategies increases with the extent of reopening in peer countries. This could suggest that a late and slow reopening strategy could help contain 

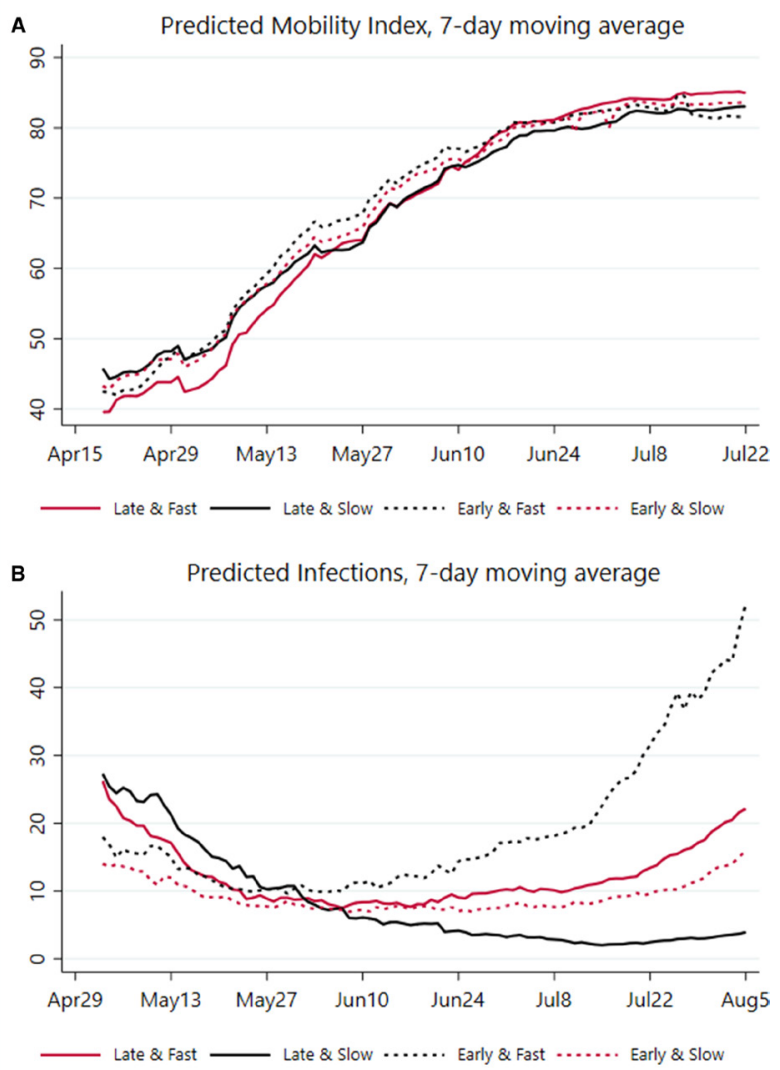

Figure 6 The graph in the top panel A shows the predicted path of the mobility index based on estimating the effect of reopening for each type of strategy. The bottom panel B shows similar predicted paths for daily cases.

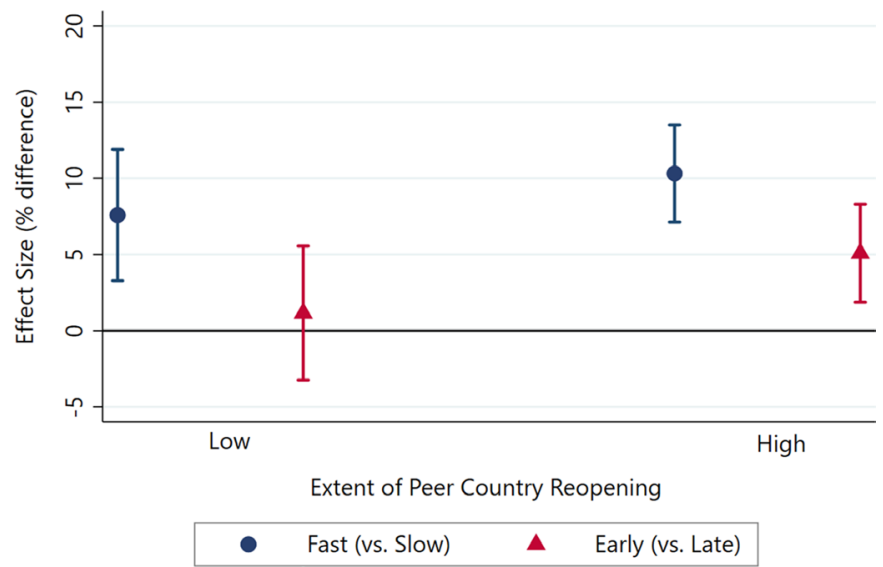

Figure 7 The graph shows the percentage difference in daily cases (21-day horizon) between fast and slow reopeners (blue dot with $90 \% \mathrm{Cl}$ line) and early versus late reopeners (red triangle with $90 \% \mathrm{Cl}$ line), depending on whether there was a low level of reopening in peer countries (one-third or less of the economy reopened) or high level of reopening in peer countries (more that one-third reopened).

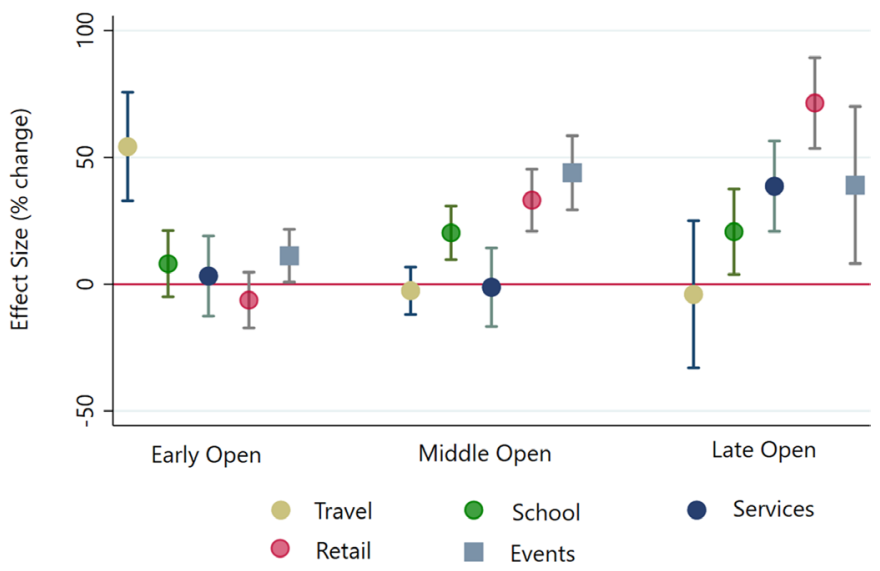

Figure 8 The graph shows the total effect (in percentage change) on daily cases from a one-unit easing of sectoral restrictions based on whether it was sequenced to open early (few other sectors opened), in the middle or late (most other sectors opened). Industry is omitted as it was always sequenced early.

the risk of infections when other countries have already reopened.

Does the sequencing of sectoral reopenings influence subsequent infections?

As documented earlier, we found substantial heterogeneity in the effect of reopening on infection across different sectors. Further, the variance decomposition of infections based on the relative contribution of sectoral reopenings suggested that opening of public places and events played an important role (table 2): they account for one of the largest shares of explained variation attributable to policies (around 3\%). To assess how the effect of each sector-specific reopening on subsequent infections varies depending on its sequencing within the countryspecific reopening plan (eg, if it is among the first sectors to reopen), we undertake the following exercise. First, for each given sector $j$, we calculate the reopenings of all other sectors and split this variable into sequencing terciles that represent how sector $j$ opened: (1) early in the schedule when majority of other sectors were not open; (2) middle in the schedule when some sectors had opened; and (3) late in the schedule when most sectors had opened. We then re-estimate our specification, for each sector $j$, by interacting the sector-specific reopening with its sequencing in the overall reopening (see online supplemental appendix section II for further details on the methodology).

Two results from this exercise stand out (figure 8). First, the effect of a given sectoral reopening step is larger when it is sequenced towards the end of the overall reopening plan. For instance, a one-unit easing in services has a little effect on infections if it happens at an early or intermediate stage (when social interaction is still limited) but the effect is positive and statistically significant if it takes place towards the end of the plan (when a large fraction of the economy is already open). The same is true for the other sectors (with the notable exception 
of international travel). This may be because community transmission, a factor identified as amplifying risks, ${ }^{31}$ is higher at later stages of reopening. The lower and insignificant effects on infections from opening international travel at a later rather than earlier stage (figure 8) may reflect that the potential importation of infections from other countries has a negligible effect when community transmission is already high. ${ }^{32}$ This may also reflect that a sector's reopening at a later stage involves social interaction synergies with other sectors already opened, which could intensify infection spread (eg, visiting a shop alone in an early stage vs visiting a shop and the beach together at a late stage).

However, the amplification effect on infections from sequencing a reopening at a later stage (in relative terms) differs across sectors. As countries need to calibrate sectoral containment measures, this finding offers insights into sectoral strategies that could mitigate infection amplification risks. At later stages (ie, when the other sectors are mostly open), the reopening of retail and events, for instance, is associated with a relatively larger increase in daily cases compared with opening other sectors, such as schools, at a similar stage. This suggests that sequencing retail to open early and schools later carries a lower risk than the opposite strategy. The relatively low amplification effect found for schools is broadly in line with early evidence documenting that reopening schools has not resulted in a significant increase in the growth rate of infections. ${ }^{33}$ The literature has attributed this result to, among other factors, stronger social distancing practices and monitoring infrastructure in schools as well as the lower susceptibility and infectivity among younger children. Still, these results should be interpreted cautiously because the implementation of school reopening policies varied across country and time (with some countries allowing voluntary attendance and with July and August subject to varied summer vacation closures).

\section{Sensitivity analysis}

\section{Mobility effects}

Our estimates on economic activity could be biased if time-varying unobservables affect both mobility and reopening plans. Including lagged infections in our baseline specification attenuates this concern but may not be sufficient. In a robustness exercise, we exploit the sectoral variation inherent in both the mobility and policy measures to ensure our results are not affected by timevarying unobservables relating to the effect of policies on mobility. We consider two sectors for analysis: retail and workplace. More precisely, we rewrite and disaggregate the outcome variable in equation 1 by stacking mobility and reopening actions by sector and measure the average effect of a sector's reopening on its mobility. We consider two sectors for analysis: retail and workplace. We measure workplace reopenings based on reopenings in services and industry.

The results shown in the top panel of online supplemental figure 7 indicate that a sectoral easing action of one unit leads to a statistically significant increase in sectoral mobility of about $2-4$ percentage points. This suggests that the results in figure 8 are robust to the presence of time-varying unobservables.

An additional robustness exercise uses a sector-specific outcome variable, sectoral job postings from indeed.com, mapped to our six sectoral reopening measures. The results shown in the bottom panel of online supplemental figure 7 suggest that a sectoral easing action of one unit leads to a statistically significant increase in sectoral job posting index of about 0.15 percentage point after approximately 4 days. The effect on job postings is lower than the effects found for mobility, suggesting some sluggishness in labour market indicators.

Finally, we perform a sensitivity analysis to account for any seasonal biases. While our analysis incorporates flexible time effects which account for any global seasonal patterns that may be present in the Google mobility indicator, we rerun our analysis with an alternative mobility indicator, the TomTom Traffic Index, which uses traffic data in 2019 as a baseline. Our results, reported in online supplemental figure 8 , are qualitatively unchanged with only a slight difference in the magnitude of the effects, suggesting that our principal findings are robust to any seasonal effects.

\section{Infection effects}

In similar vein, our estimates on infections could be biased if the ramp-up of testing is jointly correlated with reopening policies and infection evolution. In addition, it may be that the larger effect found for countries opening faster or earlier reported in figure 4 may be reflecting increased testing capacity in those countries. To address these concerns, we run an additional specification controlling for daily tests per capita (using a smaller sample given data limitations). Online supplemental figure 9 shows the results from this exercise - we obtain similar results for both the average and differential effects of reopenings, suggesting that our results are robust to developments in testing.

In online supplemental figure 10, we also present another set of robustness results controlling further for self-reported mask usage and self-reported number of (non-household) social contacts. Both these variables capture the effect of other non-pharmaceutical interventions that could be correlated with reopening strategies and subsequent infections. It should be noted, however, that the results from this specification are based on a reduced sample of countries and time period that were included in the Imperial College survey. The results show that our baseline findings are robust to controlling for testing and the implementation of other nonpharmaceutical interventions.

Using our alternative, seasonally adjusted TomTom mobility indicator, we can extend our sample to cover the 2020 summer holiday period (July to August), just before a second round of containment measures started to be imposed. In online supplemental figure 11, we show that 
our main findings continue to hold even when the sample is extended to cover the summer holiday period. While the sensitivity of infections with respect to reopenings is higher during the holidays (see online supplemental table 4), the effect is not statistically significant and is generally imprecise, given the limited set of reopening actions taken during this period.

\section{DISCUSSION}

\section{Principal findings and their interpretation}

We find that the adoption of opening measures is associated with a significant increase in the Google mobility indicator, a high-frequency proxy for economic activity. The results suggest that a one-unit change in the reopening index (eg, moving from fully closed to partially open in one sector) is associated, on average, with an initial increase in mobility of $1-1.5$ percentage points. The effect declines gradually over time but remains statistically significant for almost 2 weeks. Our results suggest that about $30 \%$ of increased mobility is attributable to reopening measures, with proxies for the role of voluntary distancing accounting for a smaller fraction (less than 10\%). To put these results in perspective, opening all six sectors at once (from fully closed) is likely to increase the mobility by approximately 20 percentage points over a week.

We also find that, on average, reopening policies lead to an increase in subsequent infections. As expected, the effect is only statistically significant after a certain lag of about 2 weeks. A one-unit easing increases the weekly trend of new cases by $7 \%$ and $10 \%$ after 2 and 3 weeks, respectively. The effect on the weekly trend of new daily deaths is about $2 \%$ at a similar horizon. While the average effect of policy measures is statistically significant, only about $20 \%$ of the variation in subsequent infections can be attributable to reopening measures.

We then turn to assess if any lessons can be drawn from the heterogeneous strategies pursued by European countries. We find that, indeed, the average effect of reopening measures on subsequent infections masks heterogeneous effects across countries' reopening strategies.

First, we find that a given reopening measure appears to have a larger effect on subsequent infections if the country starts opening early, when the circulation of virus is still pervasive and infection rates are growing; or if the reopening plan is implemented fast, with measures not sufficiently spaced over time. This likely reflects the very non-linear nature of contagion. For instance, a given easing of restrictions is likely to lead to a larger increase in infections when many people are still infected (ie, when reopening happens early). Similarly, reopening twice as fast is likely to lead to a more than proportional increase in infections.

In contrast, the effect on mobility of a given reopening measure is not different between countries opening slow versus fast or opening late versus early. In other words, easing containment restrictions by a unit delivers similar economic effects regardless of how and when a country exits, but has differential effects on infections, with a much smaller increase in cases if reopening is pursued in a late and slow manner. These results do not mean the effect on activity is inconsequential since postponing or slowing the reopening actions implies that full reopening is delayed. But the results suggest the economic cost of gradual strategies is not disproportionately larger, while the infection risk appears to be so.

Second, we also find that the marginal effect of opening individual sectors on subsequent infections depends on the sequencing of sectoral measures-that is, on how much of the economy is already open when the sectoral measure is adopted. For most sectors (with the notable exception of travel), the effect of reopening on infections is amplified when the individual action is taken at a relatively later stage of the reopening plan (in which a larger fraction of the economy is already open). However, the amplification effect on infections from sequencing a reopening at a later stage (in relative terms) differs across sectors, being higher, for instance, for retail and events.

\section{CONCLUSION AND POLICY IMPLICATIONS}

A continuous calibration of containment policies to mitigate the adverse health impact of the pandemic may be needed, until population immunity through widespread vaccinations is achieved. This fine-tuning involves uncomfortable trade-offs: the results in the paper show that reopening measures led to a much-needed recovery in economic activity, but this came at the cost of some uptick in infections already under way at end-August.

While this result is consistent with findings on the effect of the introduction of lockdowns, the findings in the paper point to some novel dimensions of the trade-off between economic activity and spread of the pandemic during the reopening phase. Our findings suggest some merit of gradual and prudent reopening strategies.

Certainly, the overall success in dealing with the pandemic as economies fully reopen will depend on the general principles regarding the timing, pace and sequencing of measures outlined here, and, crucially, on the population's collective behaviour. Further work is needed on examining whether a targeted approach to applying containment measures as activity keeps resuming can reduce the odds of new massive outbreaks at check.

Contributors All authors (JF, CMG, BG, MP, SW) contributed equally to designing the conceptualisation and execution of the analysis, including research and data compilation. All authors contributed equally to analysing and interpreting the data and drafting and preparing the manuscript.

Funding The authors have not declared a specific grant for this research from any funding agency in the public, commercial or not-for-profit sectors.

Competing interests None declared.

Patient consent for publication Not applicable.

Ethics approval This study does not involve human participants.

Provenance and peer review Not commissioned; externally peer reviewed. 
Data availability statement Data are available in a public, open access repository. Our database on reopening is provided at Harvard Dataverse: https://doi.org/10. 7910/DVN/WIGMKX.

Supplemental material This content has been supplied by the author(s). It has not been vetted by BMJ Publishing Group Limited (BMJ) and may not have been peer-reviewed. Any opinions or recommendations discussed are solely those of the author(s) and are not endorsed by BMJ. BMJ disclaims all liability and responsibility arising from any reliance placed on the content. Where the content includes any translated material, BMJ does not warrant the accuracy and reliability of the translations (including but not limited to local regulations, clinical guidelines, terminology, drug names and drug dosages), and is not responsible for any error and/or omissions arising from translation and adaptation or otherwise.

Open access This is an open access article distributed in accordance with the Creative Commons Attribution Non Commercial (CC BY-NC 4.0) license, which permits others to distribute, remix, adapt, build upon this work non-commercially, and license their derivative works on different terms, provided the original work is properly cited, appropriate credit is given, any changes made indicated, and the use is non-commercial. See: http://creativecommons.org/licenses/by-nc/4.0/.

Author note The views expressed in this paper are those of the author(s) and do not necessarily represent the views of the IMF, its Executive Board, or IMF management.

ORCID iD

Manasa Patnam http://orcid.org/0000-0002-4335-2095

\section{REFERENCES}

1 Caselli F, Grigoli F, Lian W. The great Lock- down: dissecting the economic effects. World Economic Outlook, 2020.

2 von der Leyen U, Michel C. Joint European roadmap towards lifting COVID-19 containment measures. communication. Brussels, Belgium: European Commission, 2020.

3 Islam N, Sharp SJ, Chowell G, et al. Physical distancing interventions and incidence of coronavirus disease 2019: natural experiment in 149 countries. BMJ 2020;370:m2743.

4 Salvatore M, Basu D, Ray D, et al. Comprehensive public health evaluation of lockdown as a non-pharmaceutical intervention on COVID-19 spread in India: national trends masking state-level variations. BMJ Open 2020;10:e041778.

5 Coccia M. Different effects of lockdown on public health and economy of countries: results from first wave of the COVID-19 pandemic. Journal of Economics Library 2021;8:45-63.

6 Islamaj ET, Mattoo, A D. Lives versus livelihoods during the COVID-19 pandemic: how testing softens the trade-off. Covid Economics 2021;82.

7 Bodenstein M, Corsetti G, Guerrieri L. Economics and epidemiological effects of mandated and spontaneous social distancing. Covid Economics 2021;71.

8 Augustine J, Hourani K, Molla R. Economy versus the disease: reopening mechanisms for COVID-19. Physics and Society 2020

9 Touhidul Islam ASM. Reopening the economy under the threat of COVID-19 resurgence. IUP Journal of Operations Management 2020;19:7-29.

10 Bowie C, Hill T. Exit strategy to control COVID-19 and relaunch the economy. BMJ 2020;369:m1851.

11 Han E, Tan MMJ, Turk E, et al. Lessons learnt from easing COVID-19 restrictions: an analysis of countries and regions in Asia Pacific and Europe. The Lancet 2020;396:1525-34.
12 Ruktanonchai NW, Floyd JR, Lai S, et al. Assessing the impact of coordinated COVID-19 exit strategies across Europe. Science 2020;369:1465-70.

13 Gupta S, Simon K, Wing C. Mandated and voluntary social distancing during the COVID-19 epidemic. Brookings Papers on Economic Activity, 2020.

14 Cheng W, Carlin P, Carroll J. Back to business and (re) employing workers? labor market activity during state COVID- 19 Reopenings. NBER working paper, 2020.

15 Glaeser EL, Jin GZ, Leyden BT. Learning from deregulation: the asymmetric impact of Lockdown and reopening on risky behavior during COVID-19. NBER working paper, 2020.

16 Yan Y, Malik AA, Bayham J, et al. Measuring voluntary and Policyinduced social distancing behavior during the COVID-19 pandemic. Proc Natl Acad Sci U S A 2021;118:e2008814118.

17 Li Y, Campbell H, Kulkarni D, et al. The temporal association of introducing and lifting non-pharmaceutical interventions with the time-varying reproduction number (R) of SARS-CoV-2: a modelling study across 131 countries. Lancet Infect Dis 2021;21:193-202.

18 Franks J, Gruss B, Mulas Granados C. "Data for: Reopening After Lockdowns: Evidence from Europe's First Wave of COVID Infections", 2021. Available: https://doi.org/10.7910/DVN/WIGMKX [Accessed 15 Sep 2020].

19 Hale T, Petherick A, Phillips T. Variation in government responses to COVID-19. Blavatnik school of government working paper, 2020. Available: https://www. bsg. ox. ac. uk/ research/ research- projects/ coronavirusgovernment-response- tracker [Accessed 15 Sep 2020].

20 European Commission measures dashboard. Available: https:// covid-statistics.jrc.ec.europa.eu/RMeasures [Accessed $15 \mathrm{Sep}$ 2020].

21 ACAPS COVID-19 government measures dataset. Available: https:// www.acaps.org/covid-19-government-measures-dataset [Accessed 15 Sep 2020].

22 Google Mobility. Covid-19 community mobility reports. Available: https://www.google.com/covid19/mobility [Accessed 15 Sep 2020].

23 TomTom traffic index. Available: https://github.com/ ActiveConclusion/COVID19_mobility [Accessed 17 July 2021].

24 European centre for disease prevention and control. Available: https://www. ecdc. europa. eu/ en/ publicationsdata/downloadtodays- data- geographic- distribution- covid-19-cases-worldwide [Accessed 15 Sep 2020].

25 Our world in data. Coronavirus. Available: https://ourworldindata.org/ coronavirus [Accessed 15 Sep 2020].

26 Jones SP. Imperial College London YouGov Covid 19 behaviour Tracker. Covid data hub, 2020. Available: http://www.coviddatahub. com [Accessed 15 Sep 2020].

27 Jordà Òscar. Estimation and inference of impulse responses by local projections. Am Econ Rev 2005;95:161-82.

28 STATA codes package for local projection analysis. Available: https:// sites.google.com/site/oscarjorda/home/local-projections

29 Henderson JV, Squires T, Storeygard A, et al. The global distribution of economic activity: nature, history, and the role of Trade1. Q J Econ 2018;133:357-406.

30 Jinjarak Y, Ahmed R, Nair-Desai S. Accounting for global COVID-19 diffusion patterns, January-April 2020. National Bureau of Economic Research, 2020.

31 Stage HB, Shingleton J, Ghosh S. Shut and re-open: the role of schools in the spread of COVID-19 in Europe. arXiv preprint arXiv 2020:200614158.

$32 \mathrm{WHO}$. Public health considerations while resuming inter- national travel, 30 July 2020. who report, 2020.

33 Levinson M, Cevik M, Lipsitch M. Reopening primary schools during the pandemic. N Engl J Med Overseas Ed 2020;383:981-5. 